2. Cotugno G, Annunziata P, Tessitore A, et al. Long-term amelioration of feline mucopolysaccharidosis VI after AAV-mediated liver gene transfer. Mol Ther 2011;19: 461-469.

3. Ferla R, O'Malley T, Calcedo R, et al. Gene therapy for mucopolysaccharidosis type VI is effective in cats without pre-existing immunity to AAV8. Hum Gene Ther 2013;24: 163-169.

4. Ferla R, Claudiani P, Cotugno G, et al. Similar therapeutic efficacy between a single administration of gene therapy and multiple administrations of recombinant enzyme in a mouse model of lysosomal storage disease. Hum Gene Ther 2014; 25:609-618.

5. Tessitore A, Faella A, O'Malley T, et al. Biochemical, pathological, and skeletal improvement of mucopolysaccharidosis VI after gene transfer to liver but not to muscle. Mol Ther 2008;16:30-37.

6. Tessitore A, Pirozzi M, Auricchio A. Abnormal autophagy, ubiquitination, inflammation and apoptosis are dependent upon lysosomal storage and are useful biomarkers of mucopolysaccharidosis VI. Pathogenetics 2009; 2:4.

(c) European Union 2015. Published by Mary Ann Liebert, Inc.

DOI: 10.1089/humc.2015.2526

\section{Gene Therapy for X-Linked Chronic Granulomatous Disease (Net4CGD)}

Contract No.: 305011; EU contribution: € 5,999,607; Total costs: $€ 8,302,977.60$; Starting date: 01/12/2012; Duration: 48 months

\section{Background and Objectives}

$\mathbf{T}$ HE NET4CGD EUROPEAN CONSORTIUM is focused on the clinical development of gene therapy for patients with the $\mathrm{X}$-linked form of chronic granulomatous disease (X-CGD). CGD is a debilitating primary immunodeficiency affecting children and young adults. ${ }^{1}$ This rare inherited disorder of the phagocytes is caused by absence of NADPH oxidase activity and characterized by the inability of monocytes and neutrophils to produce reactive oxygen species in response to stimuli. The X-linked recessive form is the most frequent (65\% of cases) and is caused by mutations in the $C Y B B$ gene (encoding gp91phox). Affected patients present an elevated susceptibility to bacterial and fungal infections, as well as an excessive inflammatory response leading to granuloma formation. Invasive aspergillosis is the leading cause of death in patients with CGD. Conventional treatment of CGD consists of lifelong prophylaxis with antibiotics, antimycotics, and/or interferon gamma. To date, hematopoietic stem cell transplantation (HSCT) with a suitable donor allows permanent cure of CGD, and well-tolerated reduced intensity conditioning regimens have been established. ${ }^{2}$ However, at least one-third of patients do not have an HLA-matched compatible donor, and HSCT in these patients is associated with high morbidity and mortality. Gene therapy based on autologous HSC correction may represent a definitive cure for CGD patients for whom allogeneic HSCT is not possible. ${ }^{3}$
Several members of the Net4CGD consortium have already attempted hematopoietic gene correction of X-CGD using gp91 gammaretroviral gene transfer vectors. While functional correction and clinical benefit was initially achieved, problems arose, linked to insertional mutagenesis, vector silencing, and lack of long-term engraftment. ${ }^{1}$ A new lentiviral vector (LV) was developed to express gp91phox in myeloid cells. ${ }^{4}$ Encouraging results obtained in preclinical studies and through the compassionate treatment of a patient in London have prompted us to test the LV in a multicenter study in several European centers expert in CGD. The trial sponsor is Genethon, a nonprofit organization dedicated to the development of gene therapy in rare diseases. An orphan drug designation was obtained for autologous hematopoietic cells genetically modified with an LV containing the human gp91 (phox) gene (EU/3/12/957—EMA/OD/118/11).

The Net4CGD project is focused on the clinical development of this new orphan drug, which could rapidly become a new treatment option for patients with X-CGD. This will be achieved through the following objectives:

- Conducting a phase I/II trial in eligible X-CGD patients, with LV gene-modified autologous HSC to test the safety and efficacy of the technology

- Collecting high-quality data by conducting trials in expert institutions, testing the same LV product with harmonized procedures and protocols to facilitate product registration

- Assessing functional innate immune restoration obtained by hematopoietic gene therapy

- Obtaining large-scale "omics" and vector genome stability analysis, including bioinformatic data mining, dynamic sequence data storage, and whole integrome sequencing, to evaluate vector safety in man

\section{Approach and Methodology}

The Net4CGD consortium includes seven partner laboratories with scientific and clinical expertise, three small and medium enterprises in the biotechnological and service sector, and one partner fully dedicated to the management of the consortium. The main tasks for the consortium over the next 4 years include (1) manufacturing clinical-grade vector to support clinical studies, (2) conducting a multicenter phase I/II trial in eligible X-CGD patients, with lentiviral gene-modified autologous hematopoietic stem cells to evaluate the safety and efficacy of the product, (3) ensuring high-quality and harmonization of products and procedure to facilitate future product registration, and (4) obtaining state-of-the-art information on biological efficacy and safety in patients by assessing immune restoration and large-scale integrome data. In addition, the consortium aims to communicate effectively the results of its efforts toward the scientific community, patients, families, and the general public.

\section{Main Findings}

A European multicenter gene therapy trial for X-CGD has been successfully initiated since the start of the project, and three centers are currently open to recruit adult or pediatric patients.

A unique Investigational Medicinal Product Dossier was submitted by the sponsor to the different competent national regulatory authorities in the United Kingdom, Switzerland, and Germany. The clinical trial is registered at the European 


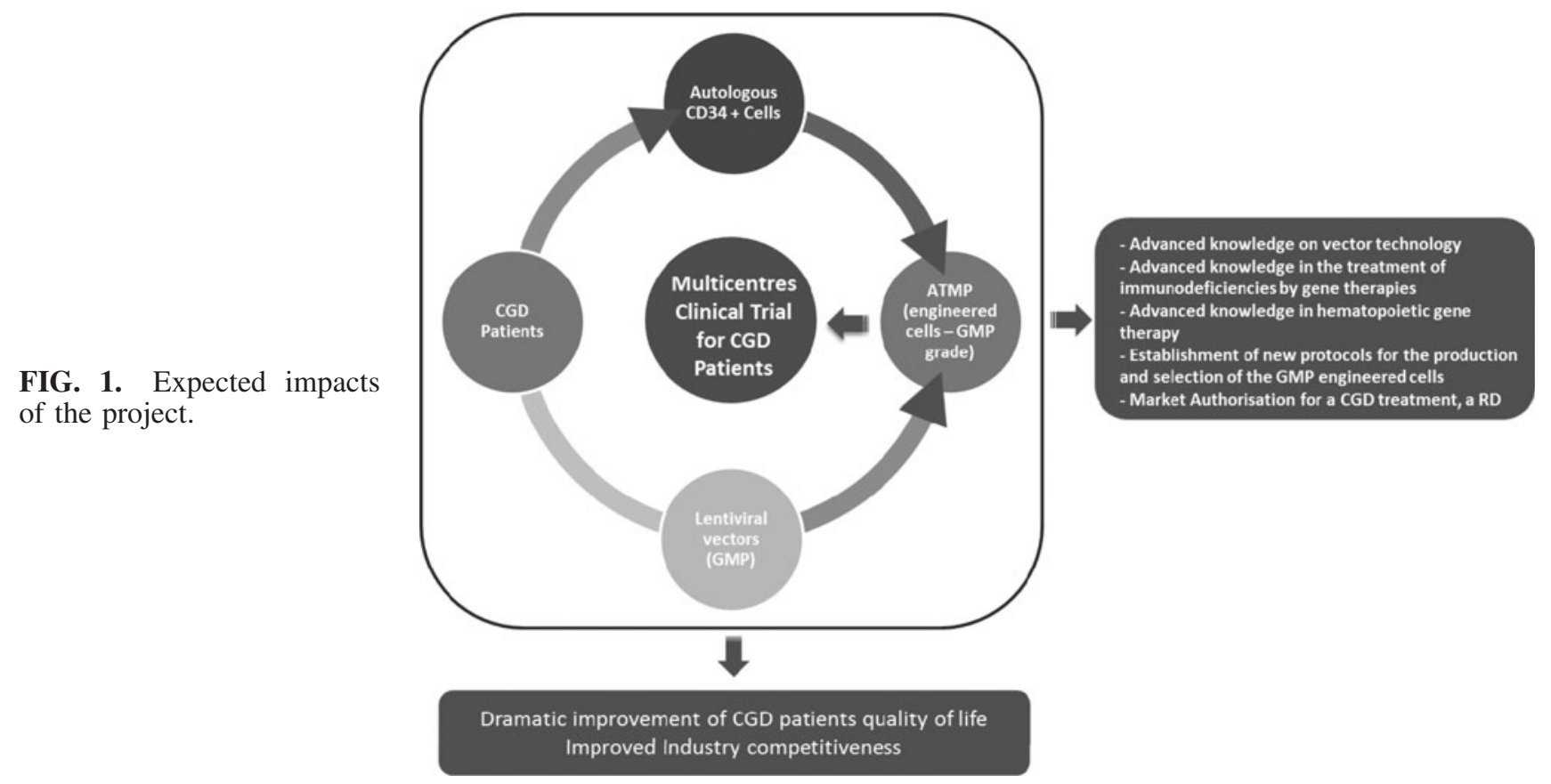

Medicines Agency under EudraCT: 2012-000242-35. The trial has been approved in the different states over a period ranging from 2013 to 2014. At present three clinical centers are opened (London, Frankfurt, and Zürich) and will enroll up to five patients each.

To conduct the trial, the consortium has developed the clinical G1XCGD vector, which is a latest-generation rHIVderived LV allowing strong expression of the transgene in myeloid cells from a chimeric promoter, ${ }^{4}$ and capable of correcting the CGD defect in phagocytes. The process for the largescale manufacture of clinical-grade G1XCGD LV has been developed and implemented at Genethon in compliance with Good Manufacturing Practice. Several lots of vector have been released for clinical use. The vector-specific quality control (QC) plan has been developed in the frame of the consortium. Ongoing developments in QC are expected to significantly reduce the costs of testing. In the first 18 months of the project, the partners of the consortium have also worked together to harmonize a protocol for the transduction of patients CD34+ cells with the G1XCGD vector. Preclinical optimization has greatly improved the quality of the transduced cell product obtained during manufacture over the course of the project to date.

An initial assessment of the risk-benefit of the clinical study, reviewed by an independent ethics expert, concluded that there is a clear benefit to conduct the clinical trial using the G1XCGD lentiviral vector to treat patients suffering from CGD and who have no treatment option. Regular ethics monitoring updates are included in the project and reported to the European Commission. An advisory board and data safety monitoring board have been assembled by the sponsor of the trial.

In parallel efforts with the trial, studies are ongoing to characterize the hematopoietic compartment in CGD, to generate models of the disease, ${ }^{5}$ to investigate mechanisms regulating transgene expression, ${ }^{6}$ and to improve the methodology for genomic insertion site analysis. The consortium meets regularly and a website has been implemented to ensure the dissemination of the work (www.net4CGD.eu).

\section{Expected Outcome}

Net4CGD represents a unique European initiative to implement advanced gene therapy through coordinated action. It is built on established European excellence in research and medicine to achieve a world-leading research program across multiple European centers. The end product is expected to be a long-lasting contribution to healthcare and tangible benefits for patients, both in terms of quality of life and also in terms of the socioeconomic burden of genetic disease. The impacts arising from Net4CGD will range from fundamental studies on advanced vector technology and hematopoietic stem cell biology, to rigorous preclinical validation of advanced gene therapies and application of multicenter European clinical studies. Furthermore, the knowledge gained will form a sustainable platform on which to build novel treatments based on similar technologies in a wider collection of inherited and acquired disease. The expected impacts of the project are schematized in Fig. 1.

\section{Major Publications}

Christian B, Kerstin BK, Anja K, et al. Generation of X-CGD cells for vector evaluation from healthy donor CD34 + HSCs by shRNA-mediated knock down of gp91phox. Mol Ther Methods Clin Dev 2014;1:Article No. 14037.

Kaufmann KB, Buning H, Galy A, et al. Gene therapy on the move. EMBO Mol Med 2013;5:1642-1661.

Muller-Kuller U, Ackermann M, Kolodziej S, et al. A minimal ubiquitous chromatin opening element (UCOE) effectively prevents silencing of juxtaposed heterologous promoters by epigenetic remodeling in multipotent and pluripotent stem cells. Nucleic Acids Res 2015;43:1577-1592.

Coordinator: Dr. Anne Galy, galy@genethon.fr

Partners: The consortium Net4CGD brings together 11 partners from 4 countries.

Anne Galy, Genethon, France. 
Adrian Thrasher, University College of London, United Kingdom.

Manuel Grez, Chemotherapeutisches Forschungsinstitut Georg Speyer Haus, Germany.

Joachim Schwaeble, University Hospital Frankfurt, Germany.

Janine Reichenbach, University of Zurich, Switzerland.

Marina Cavazzana, APHP Necker, France.

Séverine Pouillot, Genosafe, France.

Klaus Kuehlcke, Europäisches Institut für Forschung und Entwicklung von Transplantationstrategien, Germany.

Manfred Schmidt, Deutsches Krebsforschungszentrum, Germany.

Tobias Paprotka and Andreas Schreiber, GATC Biotech AG, Germany.

David Koubi, Finovatis, France.

Website: www.net4cgd.eu

\section{References}

1. Grez M, Reichenbach J, Schwable J, et al. Gene therapy of chronic granulomatous disease: the engraftment dilemma. Mol Ther 2011;19:28-35.

2. Gungor T, Teira P, Slatter M, et al. Reduced-intensity conditioning and HLA-matched haemopoietic stem-cell transplantation in patients with chronic granulomatous disease: a prospective multicentre study. Lancet 2014;383: 436-448.

3. Kaufmann KB, Buning H, Galy A, et al. Gene therapy on the move. EMBO Mol Med 2013;5:1642-1661.

4. Santilli G, Almarza E, Brendel C, et al. Biochemical correction of X-CGD by a novel chimeric promoter regulating high levels of transgene expression in myeloid cells. Mol Ther 2011;19:122-132.

5. Christian B, Kerstin BK, Anja K, et al. Generation of XCGD cells for vector evaluation from healthy donor CD34+ HSCs by shRNA-mediated knock down of gp91phox. Mol Ther Methods Clin Dev 2014; 1:Article No. 14037.

6. Müller-Kuller U, Ackermann M, Kolodziej S, et al. A minimal ubiquitous chromatin opening element (UCOE) effectively prevents silencing of juxtaposed heterologous promoters by epigenetic remodeling in multipotent and pluripotent stem cells. Nucleic Acids Res 2015;43:15771592.

(c) European Union 2015. Published by Mary Ann Liebert, Inc.

DOI: 10.1089/humc.2015.2527

\section{Consortium for Products Across Europe in Duchenne Muscular Dystrophy (SCOPE-DMD)}

Contract No.: 601573; EU contribution: € 5,999,210; Total costs: € 21,358,069; Starting date: 01/06/2013; Duration: 36 months

\section{Background and Objectives}

$\mathbf{T}$ HE GOAL OF THE SCOPE-DMD project is to utilize health research outcomes from previous EU-funded projects to perform an innovative, pivotal pediatric clinical trial to obtain market authorization for an antisense oligonucleotide (AON) treating a subset of Duchenne muscular dystrophy (DMD) patients. The results of the project will lead to (1) validation and implementation of novel outcome measures and biomarkers developed in former 6th (TREAT-NMD) and 7th (BIO-NMD) European Union's Framework Programmes, (2) a novel accelerated clinical trial design and regulatory strategy, applicable to a wide range of rare diseases, and (3) patient- and healthcare provider-derived innovative commercial strategies. The project is further building on TREAT-NMD and Prosensa's interactions with regulatory agencies, progressing the acceptability of novel biomarkers and outcome measures in neuromuscular disease (NMD), and the positioning of 2'methylphosphorothioate (2'OMePS) AONs as a single drug class for expedited development programs benefitting other subset of DMD patients.

DMD is a progressive muscle wasting disease with a prevalence of $0.32-0.52$ per 10,000 inhabitants. Worldwide around 240,000 patients suffer from DMD. The X-chromosome linked disease is caused by mutations, often deletions, in the $D M D$ gene that result in the disruption of the open reading frame of the gene, leading to a loss of dystrophin protein expression. Dystrophin is assumed to have a scaffolding function in muscle fibers, where it links the subsarcolemmal actin cytoskeleton through a complex of transmembrane proteins to the extracellular matrix. Muscle fibers lacking functional dystrophin are more prone to contraction-induced damage, which over time leads to an increase of connective tissue, replacement of muscle fibers by fat, and eventually to progressive muscle weakness and wasting. DMD boys are in most cases diagnosed by 4-5 years of age, with subclinical symptoms prior to that time. Patients become generally wheelchair dependent by 12 years of age and require assisted ventilation around the age of 18. Young DMD men typically die in the second or third decade of their life from cardiac or respiratory failure, although death may also occur in the late teens. There is currently no effective disease-modifying treatment for DMD.

Many deletions in the DMD gene result in an out-of-frame dystrophin transcript and therefore a truncated and nonfunctional dystrophin. AONs are short nucleotide chains that can bind to a selected complementary nucleotide sequence of the pre-mRNA and can induce exon skipping by blocking splicing enhancer sequences present in the exon, leading to a lack of exon recognition by the RNA splicing machinery. This results in the restoration of the open reading frame of the dystrophin mRNA, such that a truncated but functional dystrophin protein, as seen in patients with Becker muscular dystrophy (BMD), is expressed in the muscles. Based on BMD clinical experience with typically improved prognosis, it is expected that AON-treated patients will have improved muscle function due to expression of this truncated but functional dystrophin. This RNA-based approach is not gene therapy, but rather RNA modulation, as it does not manipulate or insert genetic DNA code. AON therapy can be halted by stopping the systemic administration of the compound.

The clinical trial that is part of the SCOPE-DMD project is an exploratory, open-label, dose-escalation phase Ilb study. It aims to assess the efficacy, safety, pharmacodynamics, and pharmacokinetics of weekly subcutaneous doses of PRO045 in subjects with DMD. PRO045 is a 2'OMePS RNA oligonucleotide, directed against exon 45. Nonclinical studies on PRO045 have shown its skipping efficiency and a favorable safety and pharmacokinetic profile. In patients with DMD, mutations 